\title{
Reduced expression of HSP27 following HAD-B treatment is associated with Her 2 downregulation in NIH:OVCAR-3 human ovarian cancer cells
}

\author{
KUO CHU LI ${ }^{1 *}$, KYUN HEO ${ }^{2 *}$, NITIN AMBADE ${ }^{2}$, MIN KYUNG KIM ${ }^{2}$, KYUNG-HEE KIM ${ }^{2}$, \\ BYONG CHUL YOO ${ }^{2}$ and HWA-SEUNG YOO ${ }^{1}$ \\ ${ }^{1}$ East-West Cancer Center, College of Korean Medicine, Daejeon University, Daejeon 302-869; \\ ${ }^{2}$ Research Institute, National Cancer Center, Goyang-si, Gyeonggi-do 410-769, Republic of Korea
}

Received December 19, 2013; Accepted April 10, 2015

DOI: $10.3892 / \mathrm{mmr} .2015 .3876$

\begin{abstract}
The Korean traditional medicine, HangAmDan (HAD), was developed in 1996 for use as an antitumor agent, and has since been modified to HAD-B (an altered form of HAD), in order to potentiate its therapeutic effects. In the present study, the effect of HAD-B on the proliferation and invasion of NIH:OVCAR-3 and SKOV-3 human ovarian cancer cell lines was investigated. In addition, the expression of major signal transduction molecules and changes in the proteome in these cells were measured. HAD-B treatment effectively induced a reduction in the levels of cell proliferation in serum-free conditioned media. However, unaltered levels of PARP and caspase-3 indicated that HAD-B does not reduce proliferation by inducing apoptotic cell death. Fluorescence-activated cell sorting analysis revealed no significant change in apoptosis following HAD-B treatment. Invasion assay results indicated a reduced rate of invasion following HAD-B treatment. HAD-B also influenced the expression of major signal transduction molecules; the phosphorylation of mTOR and AKT was reduced, while that of ERK was increased. Alterations in the proteomes of the two cell lines were investigated following HAD-B treatment. Among the 9 proteins with differential expression, heat-shock protein $\beta$-1 (HSP27) was downregulated in NIH:OVCAR-3 cells treated with HAD-B. The reduced expression of HSP27 was associated with human epidermal growth factor receptor 2 (Her2) downregulation in these cells. In conclusion, the results of the current proteome
\end{abstract}

Correspondence to: Professor Hwa-Seung Yoo, East-West Cancer Center, College of Korean Medicine, Daejeon University, 75 Daedeokdaero, 176 beon-gil, Seo-gu, Daejeon 302-869, Republic of Korea E-mail: altyhs@dju.kr

*Contributed equally

Key words: ovarian cancer, proteome, human epidermal growth factor receptor 2, heat-shock protein 27, HangAmDan-B assessment suggest that HAD-B has the potential to suppress the proliferation and invasion of human ovarian cancer cells. HAD-B treatment of NIH:OVCAR-3 cells suppressed HSP27 expression and was also associated with Her2 downregulation.

\section{Introduction}

In 1996, the East-West Cancer Center developed an antitumor agent known as HangAmDan (HAD) (1). Originally, this compound contained 9 antitumor oriental medicinal herbs (Table I) (1). However, it was later modified to HangAmDan-B (HAD-B; Table II), which contains 8 herbs (2). The antitumor effects and safety of HAD have been tested in in vitro and in vivo studies $(2,3)$. The toxicity of HangAmDan-B (HAD-B) in mice has been investigated over short (1-week single oral dose) and long (5-week repeated oral dose) time periods (4). No mortalities or significant differences in hematological factors were observed. HAD-B was demonstrated to be safe, nontoxic and suitable for use in studies with mice. The results of animal studies have provided preclinical evidence of the safety of HAD-B, meaning that it may be used in human studies (4). A traditional Korean medical therapy, termed Wheel Balance Therapy, also produced positive results in case studies using HAD (1). The integrative cancer treatment program, termed wheel balance cancer therapy (WBCT), incorporates traditional oriental treatments with conventional cancer treatments. The WBCT program includes anticancer diet, metabolism activation, antiangiogenic and immunoenhancing herbal therapy and, meditation and exercise (5). HAD-B inhibited the cell motility and invasiveness of NCI-H460 cells, in addition to inhibiting the activity of matrix metalloproteinase (MMP)-2 and MMP-9, in a non-cytotoxic dose-dependent manner (2). HAD-B has also been associated with the suppression of mRNA and protein expression, and the upregulation of tissue inhibitors of metalloproteinase (TIMP)-1 and TIMP-2. Reduced invasion was associated with increased tightness of tight junctions (2). In addition, HAD-B treatment led to reduced levels of claudin family members, which are involved in the control of tight junctions, and are thus important for the processes underlying paracellular transport. Tight junctions and MMPs are important targets 
associated with HAD-B-mediated suppression of invasiveness in NCI-H460 cells (2). Human umbilical vein endothelial cells exhibit significant suppression of bFGF-induced proliferation, adhesion, migration and capillary tube formation in response to treatment with water extract of HangAmDan (WEHAD). This treatment was associated with the upregulation of RAD51, RAD52 and p73, and the downregulation of pFAK. Chick chorioallantoic membranes displayed a reduction in the length of blood vessels that formed following treatment with WEHAD. A role has been suggested for WEHAD in the inhibition of angiogenesis, as a mechanism that may underlie the anticancer effects of HAD (6).

Ovarian cancer is one of the most lethal types of gynecological cancer. It was the fifth leading cause of cancer-related mortality in females in the United States in 2008 (7). Each year 125,000 mortalities occur due to ovarian cancer and 204,000 new cases are diagnosed worldwide (8). Advanced disease at the time of diagnosis is one of the primary reasons for the high fatality rate; $70 \%$ females with ovarian cancer are diagnosed at an advanced stage $(7,8)$. When the disease is confined to the ovary, the rate of successful treatment is high (70-90\%). However, when diagnosis occurs at an advanced stage, the five-year survival rates drop to $20-30 \%$. In view of these statistics, early detection may be the most effective method for reducing the mortality rate of ovarian cancer (8). Ovarian cancer is the second most common type of gynecological cancer in Korea. Mortality rates for ovarian cancer increased 9.5-fold in Korea between 1983 and 2006 (9).

Heat-shock protein 27 (HSP27) has a low molecular weight and is produced in response to pathophysiological stress in animal cells (10). Malignant tumors have been demonstrated to express higher levels of HSP27 than benign tumors (11). HSP27 is an independent marker for survival in ovarian cancer (10). In a study by Elpek et al (12), it was demonstrated that HSP70 and HSP90 expression had no significant prognostic association with epithelial ovarian carcinoma, while HSP27 expression and The International Federation of Gynecology and Obstetrics (FIGO) staging were reliable indicators of prognosis. Increased levels of auto-antibodies against HSP27 in patients with breast, ovarian or endometrial cancer suggest the presence and increased levels of HSP27 in the circulation of these individuals (13). HSP27 has been observed to be exclusively localized to the cytoplasm, and is associated with high-grade tumors (14). Heat-shock proteins are important in a number of mechanisms underlying carcinogenesis and are associated with resistance to anticancer drug therapy in ovarian carcinoma (15). A previous study demonstrated that upregulation of HSP27 in human breast cancer cells reduced susceptibility to herceptin by increasing the stability of the human epidermal growth factor receptor 2 (Her2) protein (16).

In the present study, the effects of HAD-B treatment on ovarian cancer cell proliferation, and the possible mechanisms underlying the effects of HAD-B in this process were investigated.

\section{Materials and methods}

Preparation of HangAmDan-B. HAD-B (Daejeon Oriental Hospital, Daejeon, Korea), a herbal formula consisting of Radix P. notoginseng, C. militaris, C. appendiculata, Radix P. ginseng, calculus bovis, I. batatas Margarita,
Table I. Ingredients of HangAmDan.

\begin{tabular}{lc}
\hline Herbs (Latin Botanical Name) & Relative quantity (mg) \\
\hline Coix Lachryma semen & 259.0 \\
Panax notoginseng radix & 86.0 \\
Hippocampus kelloggi & 26.0 \\
Cordyceps militaris & 26.0 \\
Santsigu tuber & 26.0 \\
Ginseng radix & 26.0 \\
Bovis calculus & 17.0 \\
Ipomoea batatas Margarita & 17.0 \\
Moschus & 17.0 \\
Total quantity (1 capsule) & 500.0 \\
\hline
\end{tabular}

Table II. Ingredients of HangAmDan-B.

\begin{tabular}{lc}
\hline Herbs (Latin Botanical Name) & Relative quantity (mg) \\
\hline Panax notoginseng radix & 95.2 \\
Cordyceps Militaris & 71.4 \\
Cremastrae appendiculata Tuber & 71.4 \\
Panx ginseng radix & 71.4 \\
Bovis calculus & 47.6 \\
Ipomoea batatas Margarita & 47.6 \\
Boswellia cateri & 47.6 \\
Commiphora myrrha & 47.6 \\
Total quantity (1 capsule) & 499.8 \\
\hline
\end{tabular}

B. carteri and $C$. myrrha in powder form, was prepared as reported in previous studies (1-4) and stored at $-20^{\circ} \mathrm{C}$ prior to use. The formula was prepared as a stock solution with a final concentration of $10 \mathrm{mg} / \mathrm{ml}$, and was diluted in media during subsequent experiments, as required.

Human ovarian cancer cell lines. The NIH:OVCAR-3 and SKOV-3 human ovarian cancer cell lines were obtained from the American Type Culture Collection (www.atcc.org).

3-[4,5-dimethylthiazol-2-yl]- 2,5-diphenyltetrazolium bromide (MTT) assay. A colorimetric MTT assay was used to assess cell proliferation following HAD-B treatment. MTT assays were performed as described in a previous study (17). Briefly, equal numbers of cells were incubated in each well in $0.2 \mathrm{ml}$ serum-free or serum-containing culture medium. Following 2 or 4 days of culture, $0.1 \mathrm{mg}$ MTT (Sigma-Aldrich, St. Louis, MO, USA) was added to each well, and incubated at $37^{\circ} \mathrm{C}$ for a further $4 \mathrm{~h}$. Plates were centrifuged at $450 \mathrm{x} \mathrm{g}$ for $5 \mathrm{~min}$ at room temperature and the medium was then removed. Dimethyl sulfoxide $(0.15 \mathrm{ml})$ was added to each well to solubilize the crystals, and plates were then read at $540 \mathrm{~nm}$ using a PowerWave HT microplate spectrophotometer (Bio-Tek Instruments Inc., Winooski, VT, USA). All experiments were performed in triplicate. 
Fluorescence-activated cell sorting (FACS) analysis. OVCAR-3 and SKOV-3 cells were treated with HAD-B. Following incubation for 48 -h, apoptosis detection was conducted using a fluorescein isothiocyanate (FITC) Annexin V Apoptosis Detection kit (cat no. 556547; BD Biosciences, San Diego, CA, USA). FACS analysis was performed using a BD FACSCalibur using CellQuest Pro software (BD Biosciences).

Invasion assay. The tumor cell invasion assay was conducted in a BD BioCoat Matrigel ${ }^{\mathrm{TM}}$ Invasion Chamber, with 24-well plates (BD Biosciences, Franklin Lakes, NJ, USA). The upper chamber was filled with $2.5 \times 10^{4}$ cells in serum-free media, which were then treated with $\mathrm{HAD}-\mathrm{B}$ for $72 \mathrm{~h}$ in an incubator maintained at $37^{\circ} \mathrm{C}$ in $5 \% \mathrm{CO}_{2}$ in a humidified atmosphere. Following treatment for $72 \mathrm{~h}$ in the upper chamber, cells were fixed and stained using Diff-Quik (cat no. 38721; Sysmex Asia Pacific Pte. Ltd., Tampines Grande, Singapore). Images were captured using an Olympus BX51 microscope (Olympus Corporation, Tokyo, Japan) at a magnification of $\mathrm{x} 100$. The number of stained cells was counted, and cell invasion was expressed as the absolute cell count from a representative image taken from the lower surface of the filter.

Western blot analysis. Western blot analysis was performed as described in a previous study (17). Briefly, cell homogenates containing equivalent amounts of protein were centrifuged at 4,000 x g, and the supernatant fractions were subjected to SDS-PAGE (Invitrogen Life Technologies, Carlsbad, CA, USA). Following electrophoresis, proteins were transferred to polyvinylidene fluoride membranes (EMD Millipore, Billerica, MA, USA) and blocked by incubation for $2 \mathrm{~h}$ at $4^{\circ} \mathrm{C}$ in $1 \%$ Tween 20 -Tris-buffered saline, containing $1.5 \%$ non-fat powdered milk (Bio-Rad Laboratories, Hercules, CA, USA) and $1 \mathrm{mM} \mathrm{MgCl}$ (Biochemicals.Inc., Gyeonggi, Republic of Korea). Membranes were incubated for $2 \mathrm{~h}$ at room temperature with primary antibodies against caspase-3 (cat. no. 9664; rabbit monoclonal; 1:1,000; Cell Signaling Technology, Inc., Danvers, MA, USA), PARP (cat. no. 9541; rabbit polyclonal; 1:1,000; Cell Signaling Technology, Inc.), HSP27 (cat. no. ab1426; rabbit polyclonal; 1:1,000' Abcam, Cambridge, UK), p-HSP27 (Ser-15; cat. no. ab5581; rabbit polyclonal; 1:1,000; Abcam), p-HSP27 (Ser-78; cat. no. ab32501; rabbit monoclonal; 1:1,000; Abcam), p-HSP27 (Ser-82; cat. no. ab90537; rabbit polyclonal; 1:1,000; Abcam), Her2 (cat. no. ab2428; rabbit polyclonal; 1:1,000; Abcam), p-AKT (Ser-473; cat. no. 9271; rabbit polyclonal; 1:1,000; Cell Signaling Technology, Inc.), AKT (cat. no. 9272; rabbit polyclonal; 1:1,000; Cell Signaling Technology, Inc.), phosphorylated mechanistic target of rapamycin (p-mTOR; Ser-2448; cat. no.2971, rabbit polyclonal; 1:1,000; Cell Signaling Technology, Inc.), mTOR (cat. no. 2972; rabbit polyclonal; 1:1,000; Cell Signaling Technology, Inc.), phosphorylated extracellular signal regulated kinases (p-ERK; cat. no. 4370; rabbit monoclonal; 1:1,000; Cell Signaling Technology, Inc.) and actin (cat. no. sc-69879; mouse monoclonal; 1:1,000; Santa Cruz Biotechnology, Inc. Dallas, TX, USA). Membranes were then washed 3 times for 15 min with blocking solution, and incubated with diluted horseradish peroxidase-conjugated secondary antibodies
(SouthernBiotech, Birmingham, AL, USA) for $1 \mathrm{~h}$ at room temperature. This was followed by 3 washes with blocking solution for $15 \mathrm{~min}$, incubation with WEST-ZOL ${ }^{\circledR}$ plus chemiluminescence reagent (Intron Biotechnology, Inc., Gyeonggi-do, Korea) for $1 \mathrm{~min}$, and exposure to film (Kodak Blue XB-1; Kodak, Rochester, NY, USA).

2-D electrophoresis (E), liquid chromatography-mass spectrometry (LC-MS)/MS and database searching. 2-DE analysis was performed as described in a previous study (17). Proteins (150 $\mathrm{mg}$ ) were applied to $13 \mathrm{~cm}$ immobilized non-linear gradient strips ( $\mathrm{pH} 3-10$; GE Healthcare, Chalfont, UK), and focused at $8,000 \mathrm{~V}$ within $3 \mathrm{~h}$. For 2-D separation, 12\% polyacrylamide gels (chemicals from Serva Electrophoresis $\mathrm{GmbH}$, Heidelberg, Germany and Bio-Rad Laboratories) were used. 2-DE gels were stained with a Colloidal Blue Staining kit (Invitrogen Life Technologies) for $24 \mathrm{~h}$, and then destained with deionized water. 2-DE gels containing proteins of interest were excised, destained with $50 \%$ acetonitrile in $0.1 \mathrm{M}$ ammonium bicarbonate, and dried in a Savant SpeedVac Centrifugal Evaporator (Thermo Fisher Scientific, Waltham, MA, USA). Dried gel pieces were re-swollen with $30 \mu \mathrm{l}$ sodium bicarbonate ( $25 \mathrm{mM}$; $\mathrm{pH} 8.8$ ), containing $50 \mathrm{ng}$ trypsin (Promega Corporation, Madison, WI, USA) at $37^{\circ} \mathrm{C}$ overnight. Samples were desalted using ZipTip C18 pipette tips (EMD Millipore), and dissolved in $10 \mu 12 \%$ acetonitrile in $0.1 \%$ formic acid. Analysis was performed using a LTQ XL Linear Ion Trap Mass Spectrometer system (Thermo Fisher Scientific) in the Proteomics Core, National Cancer Center (Seoul, Korea). The mass spectrometry was set for nanospray ionization (NSI) in positive mode. A syringe pump was used to introduce the calibration solution for automatic tuning and calibration of the mass spectrometer in NSI positive ion mode. Infusion of digested samples into the ionization source of the mass spectrometer was accomplished by liquid chromatographic separation. The spray voltage was set at $+1.8 \mathrm{kV}$, the temperature of the capillary was set at $200^{\circ} \mathrm{C}$, the capillary voltage was set at $+20 \mathrm{~V}$ and the tube lens voltage was set at $+100 \mathrm{~V}$. The auxiliary gas was set to 0 arb. Full scan experiments were performed to linear trap in the range $\mathrm{m} / \mathrm{z}$ 150-2000. Systematic MS/MS experiments were performed by changing the relative collision energy and monitoring the intensities of the fragment ions. All MS/MS samples were analyzed using Proteome Discoverer software, version 1.4 (Thermo Fisher Scientific). The Proteome Discoverer was set up to search the uniprot_sprot database (www.uniprot.org) and IPI human database (www.ebi.ac.uk/IPI). The database searching parameters included up to two missed cleavages allowing for full trypsin digestion, fixed modification for carbamidomethyl cysteine and variable modifications for methionine oxidation.

Statistical analysis. Differneces between groups were calculated using Studen's t-test. $\mathrm{P}<0.05$ was considered to indicate a statistically significant difference.

\section{Results}

Suppression of the rate of proliferation following $H A D-B$ treatment in the absence of serum. In order to study the 
A
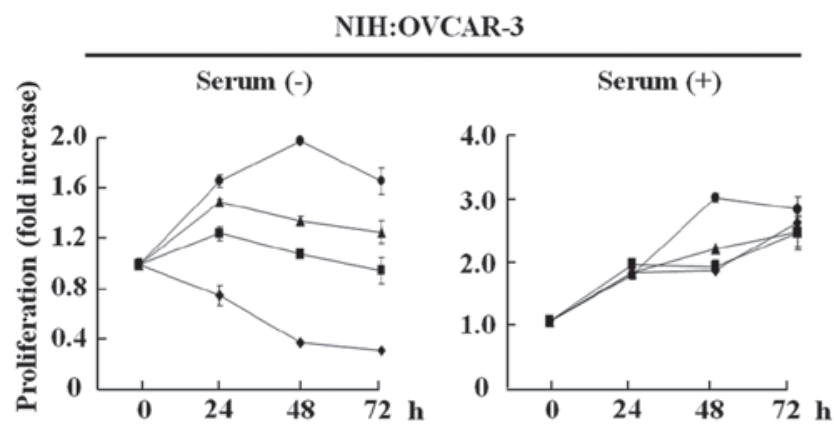

B
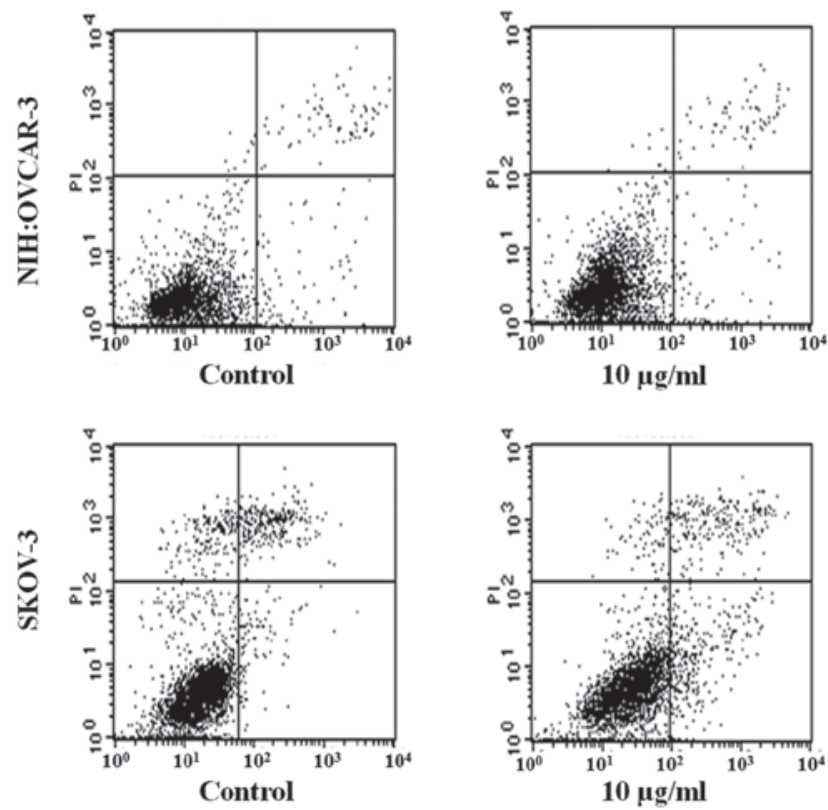

C

NIH:OVCAR-3

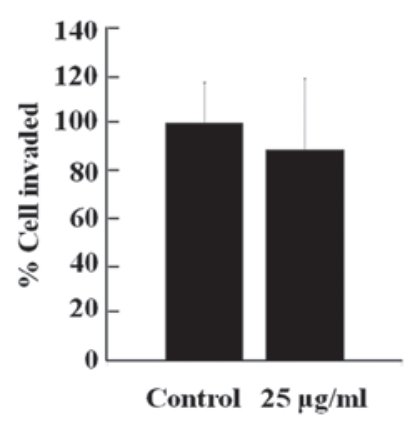

SKOV-3

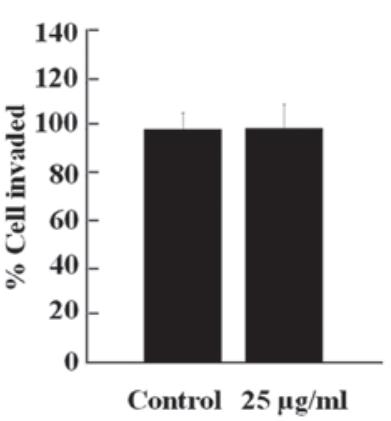

SKOV-3

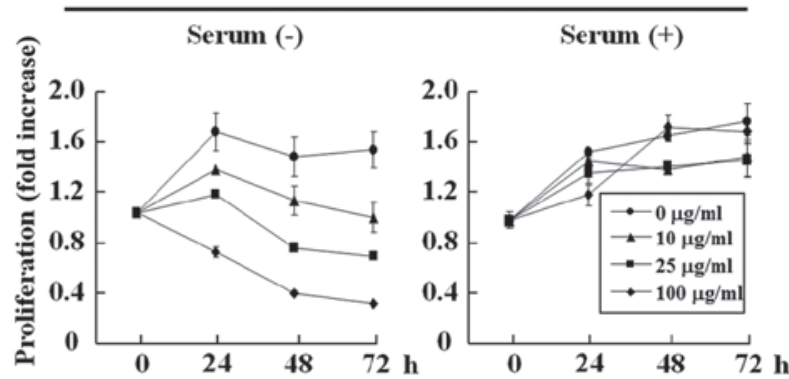

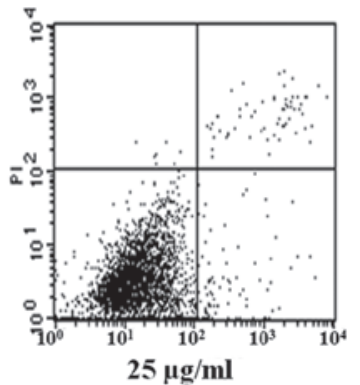
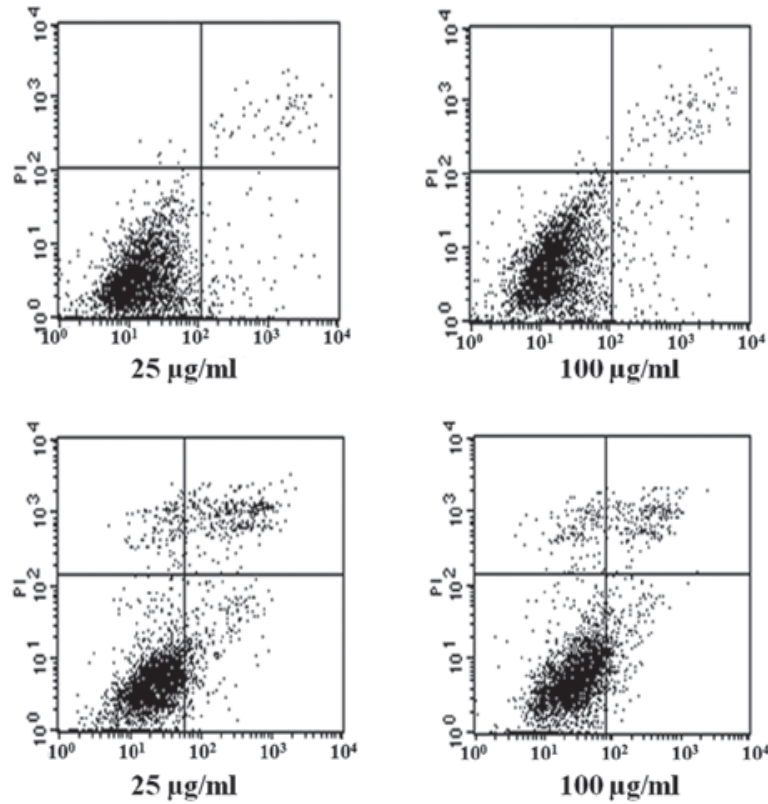

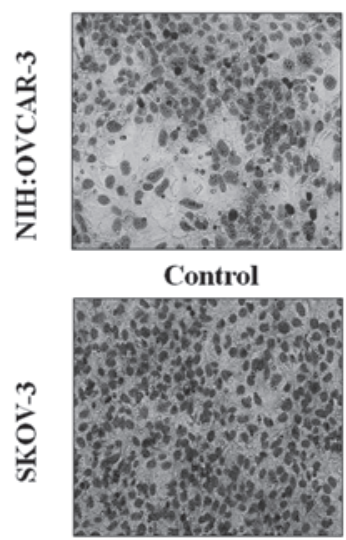

Control

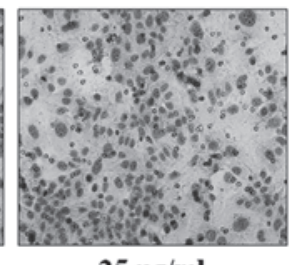

$25 \mu \mathrm{g} / \mathrm{ml}$

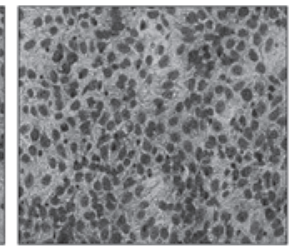

$25 \mu \mathrm{g} / \mathrm{ml}$

Figure 1. Proliferation and invasion of NIH:OVCAR-3 and SKOV-3 cell lines following HAD-B treatment. (A) Cell proliferation was suppressed following HAD-B treatment in the absence of serum. The proliferation of NIH:OVCAR-3 and SKOV-3 cells decreased in a dose- and time-dependent manner in the absence of serum. However, no significant change in proliferation was observed in response to varying drug concentrations in the presence of serum. (B) No significant change in apoptosis following HAD-B treatment was observed. The OVCAR-3 cell population moved slightly towards the apoptotic region as the HAD-B dose was increased, while SKOV-3 cells did not exhibit any alteration. (C) Suppressed invasion of OVCAR-3 cells following HAD-B treatment. The rate of invasion was slightly reduced in NIH:OVCAR-3 cells following HAD-B treatment. However, this reduction was not observed in SKOV-3 cells following treatment with HAD-B. HAD-B, HangAmDan-B.

effect of HAD-B on NIH:OVCAR-3 and SKOV-3 cell lines, an MTT assay was performed (Fig. 1A). In the absence of serum,
HAD-B treatment led to a dose-dependent reduction in the proliferation rate. With continued treatment, the rate of prolifer- 

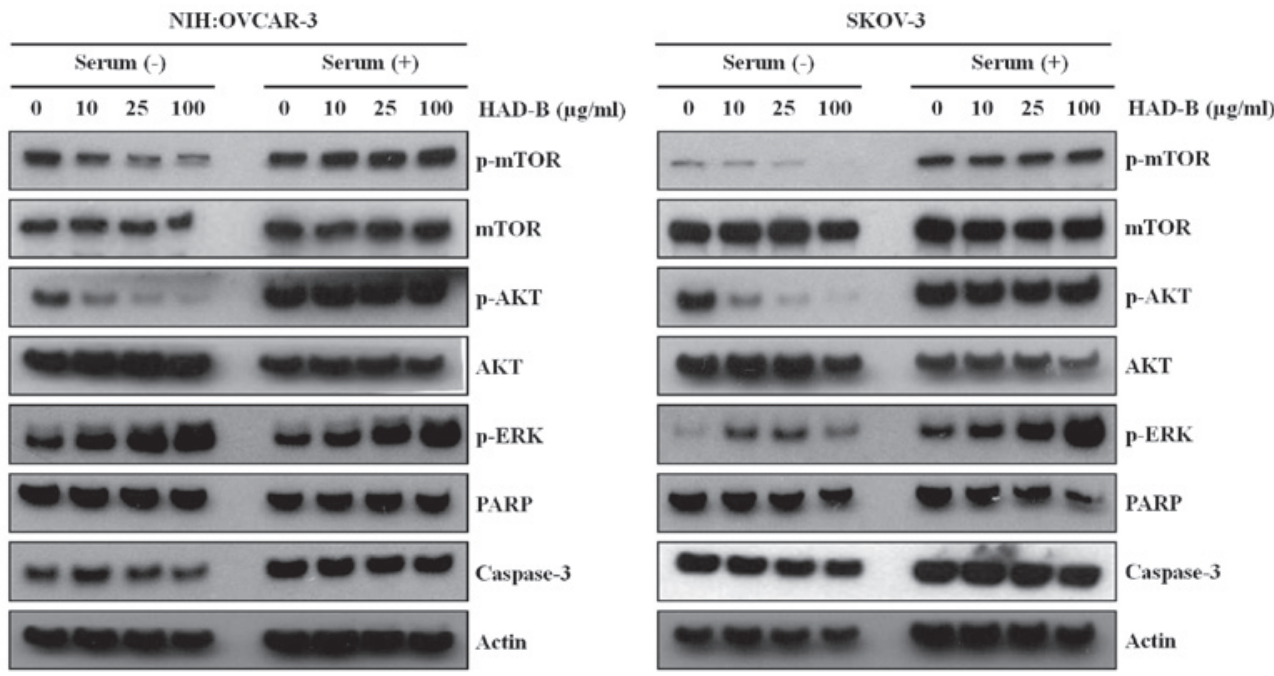

Figure 2. Reduction in the phosphorylation of signaling molecules in NIH:OVCAR-3 and SKOV-3 cell line, following HAD-B treatment. In the absence of serum, a dose-dependent reduction in phosphorylation of mTOR and AKT was observed, while a dose-dependent increase in the phosphorylation of ERK occurred, following HAD-B treatment. No significant alteration was observed in phosphorylation in the presence of serum. HAD-B, HangAmDan-B; mTOR, mechanistic target of rapamycin; AKT, protein kinase B; ERK, extracellular signal-regulated kinases.
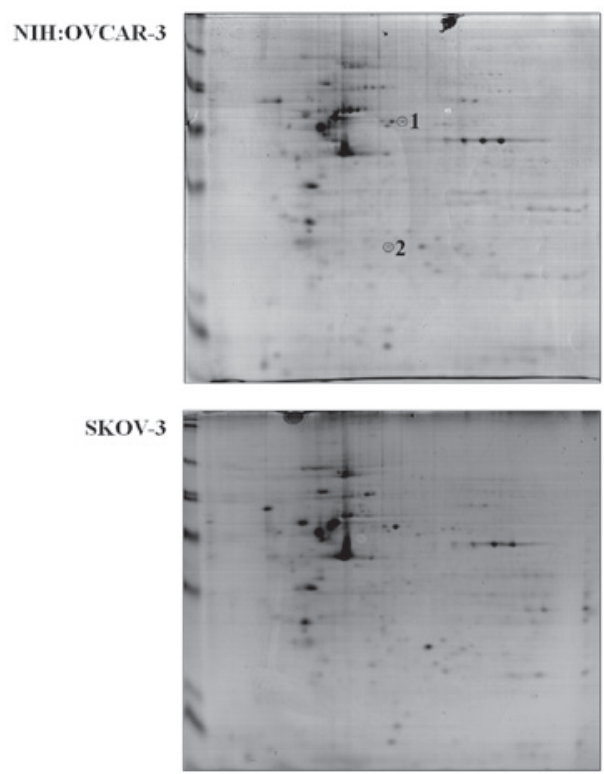

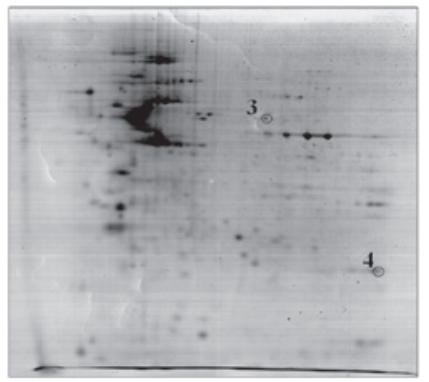

NIH:OVCAR-3 HAD-B $25 \mu \mathrm{g} / \mathrm{ml}$

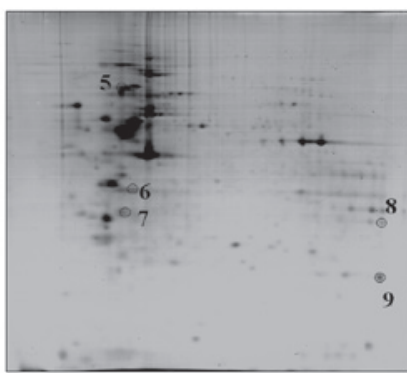

SKOV-3 HAD-B $25 \mu \mathrm{g} / \mathrm{ml}$

Figure 3. Proteome alteration in ovarian cancer cells following HAD-B treatment. Change in the expression of proteins following HAD-B treatment of NIH:OVCAR-3 and SKOV-3 cell lines. 2-D gel electrophoresis analysis indicated that 9 proteins were altered by HAD-B treatment. HAD-B, HangAmDan-B.

ation decreased in a time-dependent manner. In the presence of serum, NIH:OVCAR-3 and SKOV-3 cell lines exhibited variable changes in cell proliferation rates (Fig. 1A)

Shift in the OVCAR-3 cell population from the viable region to the apoptotic region. FACS analysis (Fig. 1B) indicated that there was a movement of a small population of OVCAR-3 cells from the viable region towards the apoptotic region. However, significant changes were not observed in apoptotic response or in the viable cell population density of OVCAR 3 cells. SKOV-3 cells did not exhibit a significant change in the proportion of cells in early or late apoptosis, following treatment with varying concentrations of HAD-B. The viable cell count was not altered significantly in the SKOV-3 cell line.
Reduced Matrigel invasion of OVCAR-3 cells following treatment with $H A D-B$. An in vitro invasion assay (Fig. 1C) using Matrigel indicated that the invasion rate remained unaltere in the NIH:OVCAR-3 and SKOV-3 cells following treatment with HAD-B.

Reduction in the phosphorylation rate of signaling molecules affecting proliferation, following $H A D$-B treatment. In order to examine the expression patterns of different signaling molecules following HAD-B treatment, western blot analysis was conducted in NIH:OVCAR-3 and SKOV-3 cell lines, in the presence and absence of serum (Fig. 2). In the absence of serum, NIH:OVCAR-3 and SKOV-3 cell lines exhibited a reduction in the phosphorylation of mTOR and AKT, in 
Table III. Identification of nine proteins by matrix-assisted laser desorption/ionization-time of flight analysis.

\begin{tabular}{llccccc}
\hline $\begin{array}{l}\text { Spot } \\
\text { number }\end{array}$ & \multicolumn{1}{c}{ Protein name } & $\begin{array}{c}\text { Accession } \\
\text { number }\end{array}$ & Score & $\begin{array}{c}\text { Sequence } \\
\text { coverage }(\%)\end{array}$ & $\begin{array}{c}\text { Molecular } \\
\text { weight (kDa) }\end{array}$ & Calculated pI \\
\hline 1 & Protein disulfide-isomerase A3 & P30101 & 30.08 & 13.47 & 56.7 & 6.35 \\
2 & Heat shock protein $\beta-1$ & P04792 & 23.42 & 30.24 & 22.8 & 6.40 \\
3 & T-complex protein 1 subunit $\beta$ & P78371 & 21.56 & 11.03 & 57.5 & 6.46 \\
4 & Peroxiredoxin-1 & Q06830 & 25.58 & 18.59 & 22.1 & 8.13 \\
5 & 78 kDa glucose-regulated protein & P11021 & 110.68 & 32.87 & 72.3 & 5.16 \\
6 & Glyceraldehyde-3-phosphate & P04406 & 30.54 & 19.40 & 36.0 & 8.46 \\
7 & dehydrogenase & P08758 & 33.87 & 24.38 & 35.9 & 5.05 \\
8 & Annexin A5 & P21796 & 44.58 & 33.92 & 30.8 & 8.54 \\
9 & Voltage-dependent anion-selective & & & & & 8.13 \\
\hline
\end{tabular}

pI, isoelectric point.

A

NIH:OVCAR-3

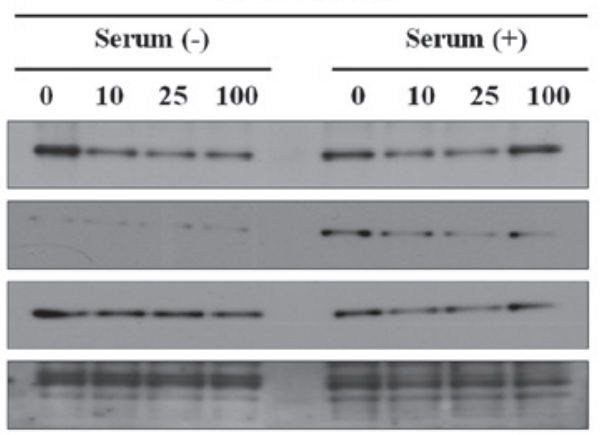

SKOV-3

\begin{tabular}{|c|c|c|c|c|c|c|c|}
\hline \multicolumn{3}{|c|}{ Serum (-) } & \multicolumn{4}{|c|}{ Serum $(+)$} & \multirow[b]{2}{*}{ HAD-B $(\mu \mathrm{g} / \mathrm{ml})$} \\
\hline $\mathbf{0}$ & $10 \quad 25$ & 100 & 0 & 10 & 25 & 100 & \\
\hline - & - & & - & & & - & HSP27 \\
\hline & & 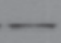 & 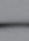 & & & & p-HSP27 (Ser-15) \\
\hline & & 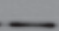 & 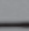 & - & . & - & p-HSP27 (Ser-78) \\
\hline & 5 & & & & & & p-HSP27 (Ser-82) \\
\hline
\end{tabular}

B

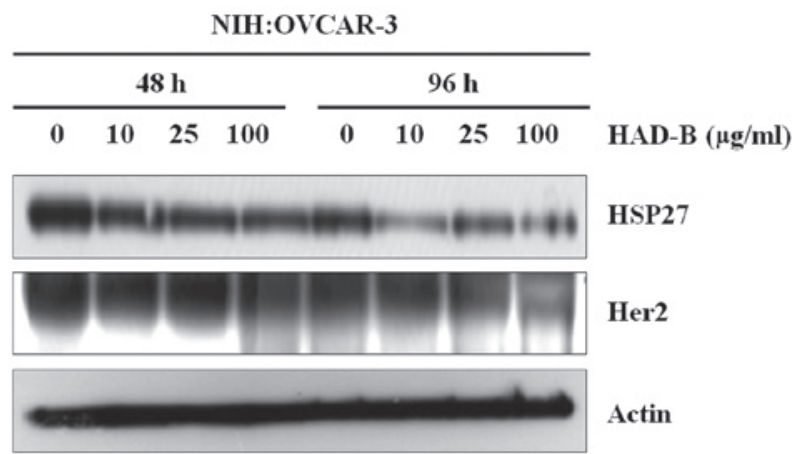

Figure 4. HSP27 and Her2 were suppressed by HAD-B treatment. (A) HSP27 and p-HSP27 expression in NIH:OVCAR-3 and SKOV-3 cell lines. Reduced levels of HSP27 and p-HSP27 in NIH:OVCAR-3 cells were observed. No significant reduction in HSP27 levels in SKOV-3 cells was detected. (B) Association between Her2 and HSP27 expression. Her2 suppression was associated with a reduction in HSP27 levels in a dose- and time-dependent manner. HSP27, heat shock protein 27; p-HSP27, phosphorylated-HSP27; Her2, human epidermal growth factor receptor 2; HAD-B, HangAmDan-B.

a dose-dependent manner. However, the phosphorylation of ERK was not suppressed. In the presence of serum, the NIH:OVCAR-3 cells exhibited no reduction in the expression of mTOR or AKT. The expression of PARP and caspase- 3 was not affected by any of the tested variables, which indicates that there was no change in the level of apoptosis in NIH:OVCAR-3 and SKOV-3 cell lines following HAD-B treatment.
Proteome alteration in ovarian cancer cells following $H A D-B$ treatment. Proteomic profiling by 2D gel electrophoresis followed by LC-MS/MS, was conducted in order to investigate proteins affected by HAD-B treatment in ovarian cancer cells (Fig. 3). Nine proteins were altered by HAD-B treatment, as described in Table III. A previous study suggested that heat-shock protein $\beta$-1, also known as HSP27, 
reduces susceptibility to herceptin in human breast cancer cells by increasing the stability of the Her2 protein.

HSP27 and Her2 are suppressed by HAD-B treatment. In order to investigate the effect of HAD-B treatment on HSP27 and p-HSP27 expression levels in NIH:OVCAR-3 and SKOV-3 cell lines, western blotting was performed in the presence and absence of serum (Fig. 4A). The results demonstrated that HAD-B reduced HSP27 and p-HSP27 levels in NIH:OVCAR-3 cells in a dose-dependent manner in the presence of serum. The expression of p-HSP27 (Ser-15) was also reduced in the absence of serum in these cells. However, no significant reduction of HSP27 levels was observed in SKOV-3 cells. In order to examine the association between the expression of HSP27 and Her2, a further western blot assay was performed (Fig. 4B), which indicated that Her2 expression was suppressed in association with reduced HSP27 levels in a dose-dependent manner, and that this reduction was more marked following longer duration of treatment.

\section{Discussion}

HAD-B has been shown to exhibit anticancer effects, including the inhibition of cell motility and angiogenesis, with consequent reduced invasiveness in cancer cell lines $(2,6)$. It is a potential novel anticancer agent with minimal or no side effects when administered within the therapeutic dose limits (4). Previous studies have demonstrated that a reduction in HSP27 expression is associated with a more advanced stage of disease, and that HSP27 is an independent marker of survival in ovarian cancer (10).

In order to investigate the effect of HAD-B on cell proliferation in NIH:OVCAR-3 and SKOV-3 cell lines, an MTT assay was performed. Following treatment with HAD-B, a suppression in the rate of proliferation was observed in the absence of serum, in a dose- and time-dependent manner. No significant reduction in the rate of proliferation was observed in the presence of serum following treatment with HAD-B. This suggested that a factor that is present in serum may interact with HAD-B or with a separate molecule, which inhibits HAD-B. Apoptosis was examined by FACS analysis, using Annexin V. The OVCAR-3 cell population shifted slightly from the viable to apoptotic region, as the HAD-B dose was increased. SKOV-3 cells displayed no alterations in viable or apoptotic cell density, or a shift in the cell population. Despite the fact that there was only a small alteration in apoptosis, the Matrigel in vitro invasion assay indicated a reduction in the invasive properties of OVCAR-3 cells. By contrast, there was no change in the invasive capability of SKOV-3 cells.

In the current study the expression profiles of various signaling molecules in NIH:OVCAR-3 and SKOV-3 cell lines following treatment with HAD-B, indicated a reduction in the phosphorylation of mTOR and AKT in the absence of serum, in a dose-dependent manner, which affected the proliferation rate following HAD-B treatment. A previous study demonstrated that inhibition of ovarian cancer cell proliferation is due to interference with the EGFR-AKT pathway (18). The HIV protease inhibitor, ritonavir, has been shown to induce cell cycle arrest and apoptosis in the MDH-2774 and SKOV-3 ovarian cell lines, in a dose-dependent manner. Ritonavir treatment resulted in $\mathrm{G}_{1}$ cell cycle arrest in ovarian cancer cells by downregulation of RB phosphorylation levels and diminution of $\mathrm{G}_{1}$ cyclins and cyclin-dependent kinases, and augmentation of their inhibitors (19). Ritonavir has also been shown to suppress the level of phosphorylated AKT in a dose-dependent manner. Administration of an AKT inhibitor may increase the therapeutic efficacy of ritonavir (19). This indicates a role for phosphorylation in the proliferation of cells.

In the present study, no change in the expression of PARP or caspase- 3 was identified, which indicates that there was no increase in apoptosis in the NIH:OVCAR-3 and SKOV-3 cell lines following treatment with HAD-B, and that HAD-B reduced proliferation levels without inducing apoptosis.

Proteomic profiling demonstrated the suppression of one protein and the increased expression of eight proteins following treatment with HAD-B. A further western blot assay indicated that HAD-B successfully reduced levels of HSP27 in NIH:OVCAR-3 cells in a dose-dependent manner in the presence of serum. No significant reduction was observed in the levels of HSP27 in SKOV-3 cells, while in OVCAR-3 cells, Her2 expression was suppressed, and HSP27 levels were reduced in a dose- and time-dependent manner. This suggests that NIH:OVCAR-3 cells are sensitive to HAD-B treatment. It has previously been demonstrated that the growth arrest and senescence of HER2-expressing cells are associated with the downregulation of HSP27 (20). In a previous study, upregulation of HSP27 in human breast cancer cells was demonstrated to reduce susceptibility to herceptin by increasing Her2 protein stability (16). These observations suggest that the reduced cell proliferation of the OVCAR-3 cell population following HAD-B treatment, was associated with a reduction in levels of HSP27, which is also associated with Her2 levels, as demonstrated by the concomitant reduction in HSP27 levels and Her2 expression. By contrast, no significant reduction was detected in the levels of HSP27 in SKOV-3 cells, following treatment with HAD-B, which also explains the absence of a change in the invasiveness of SKOV-3 cells.

In conclusion, HAD-B treatment suppressed the rate of proliferation of NIH:OVCAR-3 and SKOV-3 cell lines in the absence of serum, and the mechanism underlying this effect did not appear to involve the induction of apoptosis. HAD-B treatment was associated with limited alteration in the proteome. Notably, HSP27 was suppressed by HAD-B treatment in the absence of serum. Reduced levels of HSP27 were associated with a reduction in Her2 expression, which ultimately resulted in the inhibition of proliferation in a dose- and time-dependent manner. The reduction in invasion observed in OVCAR-3 cells was associated with a reduction in HSP27 levels following HAD-B treatment. HAD-B also inhibited phosphorylation of the signaling molecules, mTOR and AKT, which is likely to have contributed to the observed reduced rate of proliferation.

\section{References}

1. Jeong TY, Park BK, Lee YW, Cho CK and Yoo HS: Prospective analysis on survival outcomes of nonsmall cell lung cancer over IIIb treated with HangAm-Dan. Zhongguo Fei Ai Za Zhi 13: 1009-1015, 2010

2. Choi YJ, Shin DY, Lee YW, et al: Inhibition of cell motility and invasion by HangAmDan-B in NCI-H460 human non-small cell lung cancer cells. Oncol Rep 26: 1601-1608, 2011. 
3. Yu T, Moh SH, Kim SB, et al: HangAmDan-B, an ethnomedicinal herbal mixture, suppresses inflammatory responses by inhibiting Syk/NF-кB and JNK/ATF-2 pathways. J Med Food 16: 56-65, 2013.

4. Yoo HS, Lee HJ, Kim JS, et al: A toxicological study of HangAmDan-B in mice. J Acupunct Meridian Stud 4: 54-60, 2011.

5. Park HM, Kim SY, Jung IC, et al: Integrative tumor board: a case report and discussion from East-West Cancer Center. Integr Cancer Ther 9: 236-245, 2010.

6. Bang JY, Kim KS, Kim EY, et al: Anti-angiogenic effects of the water extract of HangAmDan (WEHAD), a Korean traditional medicine. Sci China Life Sci 54: 248-254, 2011.

7. Zerbini LF, Tamura RE, Correa RG, et al: Combinatorial effect of non-steroidal anti-inflammatory drugs and NF- $\kappa \mathrm{B}$ inhibitors in ovarian cancer therapy. PLoS One 6: e24285, 2011.

8. Rauh-Hain JA, Krivak TC, Del Carmen MG and Olawaiye AB Ovarian cancer screening and early detection in the general population. Rev Obstet Gynecol 4: 15-21, 2011.

9. Park B, Park S, Kim TJ, et al: Epidemiological characteristics of ovarian cancer in Korea. J Gynecol Oncol 21: 241-247, 2010

10. Geisler JP, Geisler HE, Tammela J, et al: Heat shock protein 27 an independent prognostic indicator of survival in patients with epithelial ovarian carcinoma. Gynecol Oncol 69: 14-16, 1998.

11. Langdon SP, Rabiasz GJ, Hirst GL, et al: Expression of the heat shock protein HSP27 in human ovarian cancer. Clin Cancer Res 1: 1603-1609, 1995.

12. Elpek GO, Karaveli S, Simsek T, Keles N and Aksoy NH: Expression of heat-shock proteins hsp27, hsp70 and hsp90 in malignant epithelial tumor of the ovaries. APMIS 111: 523-530, 2003.
13. De AK and Roach SE: Detection of the soluble heat shock protein 27 (hsp27) in human serum by an ELISA. J Immunoassay Immunochem 25: 159-170, 2004.

14. Elstrand MB, Kleinberg L, Kohn EC, Tropé CG and Davidson B Expression and clinical role of antiapoptotic proteins of the bag, heat shock and Bcl-2 families in effusions, primary tumors, and solid metastases in ovarian carcinoma. Int J Gynecol Pathol 28: 211-221, 2009.

15. Langmár Z and Vleskó G: The potential role of heat shock proteins in the treatment of ovarian cancer. Orv Hetil 152: 92-95, 2011 (In Hungarian).

16. Kang SH, Kang KW, Kim KH, et al: Upregulated HSP27 in human breast cancer cells reduces herceptin susceptibility by increasing Her2 protein stability. BMC Cancer 8: 286, 2008.

17. Yoo BC, Hong SH, Ku JL, et al: Galectin-3 stabilizes heterogeneous nuclear ribonucleoprotein $\mathrm{Q}$ to maintain proliferation of human colon cancer cells. Cell Mol Life Sci 66: 350-364, 2009.

18. Guo J, Schally AV, Zarandi M, Varga J and Leung PC: Antiproliferative effect of gowth hormone-releasing hormone (GHRH) antagonist on ovarian cancer cells through the EGFR-Akt pathway. Reprod Biol Endocrinol 8: 54, 2010.

19. Kumar S, Bryant CS, Chamala S, et al: Ritonavir blocks AKT signaling, activates apoptosis and inhibits migration and invasion in ovarian cancer cells. Mol Cancer 8: 26, 2009.

20. Meng L, Gabai VL and Sherman MY: Heat-shock transcription factor HSF1 has a critical role in human epidermal growth factor receptor-2 induced cellular transformation and tumorigenesis. Oncogene 29: 5204-5213, 2010. 